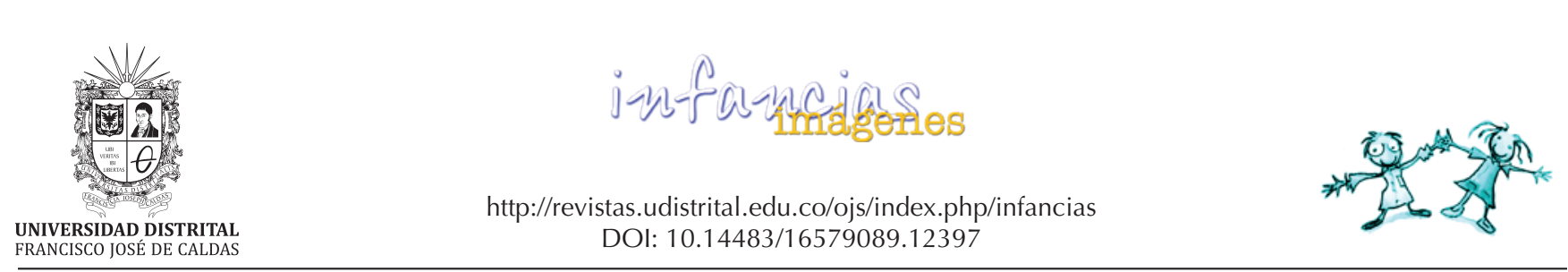

IMÁGENES DE INVESTIGACIÓN

\title{
Estado de la investigación sobre jóvenes en Bogotá, Colombia y América Latina*
}

\author{
State of Research on Youth in Bogotá, Colombia and Latin America \\ Edilberto Hernández Cano'
}

Para citar este artículo: Hernández, E. (2018). Estado de la

Recibido: 14-agosto-2017 / Aprobado: 24-abril-2018

investigación sobre jóvenes en Bogotá, Colombia y Améri-

ca Latina. Infancias Imágenes, 17(2), 185-196

\section{Resumen}

Este artículo presenta un análisis de algunos estudios que se han desarrollado sobre los jóvenes en los últimos años en Bogotá, ciertas regiones de Colombia y América Latina; además de pesquisas sobre representaciones sociales en el contexto escolar que toman como objeto las representaciones mutuas maestro-alumno. Se da razón del panorama en términos globales de los hallazgos, dificultades, tendencias, enfoques teóricos y perspectivas a la par que se indaga por los vacíos, contradicciones y tensiones. El artículo hace parte del proyecto de investigación doctoral "La construcción social de los jóvenes en su condición escolar: una mirada desde las representaciones sociales de los maestros de secundaria de Bogotá", de carácter cualitativa con enfoque en el paradigma interpretativo. La pregunta que orienta el estudio es: ¿cuáles son las representaciones sociales que han construido los maestros de educación secundaria sobre los jóvenes escolarizados en las instituciones educativas de la ciudad de Bogotá?

Palabras clave: investigación, juventud, maestro, representación social, escuela secundaria.

\section{Abstract}

This paper presents an analysis of some studies, developed on young people in recent years in Bogotá, some regions of Colombia and Latin America; in addition to inquiries about social representations in the school context that take as their object reciprocal representations between teacher-student. The view is given according global terms of findings, difficulties, trends, theoretical approaches and perspectives by inquiring gaps, contradictions and tensions. This paper is part of the doctoral research project "La construcción social de los jóvenes en su condición escolar: una mirada desde las representaciones sociales de los maestros de secundaria de Bogotá", which is a qualitative research focused in the interpretative paradigm. The guiding question is: which are the social representations constructed by high school teachers about the young people enrolled in the educational institutions of the city of Bogotá?

Keywords: research, youth, teacher, social representation, high school.

\footnotetext{
* Este artículo hace parte del estado del arte del proyecto de investigación doctoral "La construcción social de los jóvenes en su condición escolar: una mirada desde las representaciones sociales de los maestros de secundaria de Bogotá", investigación en curso en el marco del Doctorado Interinstitucional en Educación de la Universidad Distrital Francisco José de Caldas.

1 Estudiante del Doctorado Interinstitucional en Educación de la Universidad Distrital Francisco José de Caldas. Candidato a doctor, Magíster en Investigación Social Interdisciplinaria, Especialista en Gerencia de Proyectos Educativos, Licenciado en Ciencias Sociales. Docente de la Universidad Distrital Francisco José de Caldas, Docente Secretaría de Educación del Distrito. Correo electrónico: edilbertoh14@gmail.com
} 


\section{Introducción}

Se da cuenta del estado de las investigaciones sobre los jóvenes, lugar desde el que se pueden entender los procesos de construcción social de estos sujetos, así como sus diferentes modos de percibirlos y representarlos.

El proceso de construcción del conocimiento alcanzado en las investigaciones revisadas asumió la modalidad de investigación documental, la cual permitió revisar de manera detallada los documentos sometiéndolos al análisis a partir de categorías prestablecidas, con la recopilación critica de diversos estudios que abordan como objeto a los jóvenes en diferentes contextos; este ejercicio hermenéutico permitió la comprensión e interpretación de los mismos.

En resumen, se siguieron tres pasos: búsqueda de documentos, contextualización, clasificación y categorización.

En Bogotá se han elaborado algunos estudios que dan cuenta de las investigaciones sobre jóvenes en la ciudad, los cuales se analizan como fuente principal en este documento. Uno de los estudios revisados es el "Estado del arte de la investigación sobre juventud para la formulación de la política" (Serrano, Bejarano, Caicedo, Hoyos y Quintero, 2002). Este estudio fue direccionado por el departamento de investigaciones de la Universidad Central y el Departamento Administrativo de Acción Comunal del distrito, el cual se construyó a partir de la revisión de 240 referencias y 71 tesis.

Otro estudio analizado fue "Estado del arte para los campos del arte y prácticas culturales para la población de juventud en Bogotá D.C", coordinado por Martha López y direccionado por la Universidad Nacional de Colombia (2010). El objetivo central fue la realización de una revisión de las investigaciones y estudios sobre cultura y juventud en Bogotá en el período 2006-2009, así como dar cuenta del hacer de los jóvenes, de sus prácticas culturales, sus visiones de futuro y el modo en que a través de ellas reconfiguran las identidades en la perspectiva del cambio y la transformación social.

El trabajo desarrollado por Perea (2000) es muy importante para dar cuenta de los estudios sobre jóvenes en la ciudad. El autor, a partir de la categoría de identidades juveniles, desarrolla un trabajo con jóvenes de los sectores populares de la ciudad con temas centrales como las pandillas, culturas juveniles, identidades múltiples, la relación de los jóvenes con lo institucional, la escuela y la política.

También se revisa la investigación desarrollada por Edilberto Hernández (2012) sobre jóvenes y participación, en el que indaga por las representaciones sociales (en adelante RS) sobre la participación que construyen los jóvenes de la mesa local de política educativa de la localidad de Los Mártires en Bogotá.

De igual manera, los trabajos desarrollados por investigadores como Useche (2009), Amador, García y Leonel (2011), Muñoz (2011), Delgado y Arias (2008), quienes realizaron estudios y construcciones teóricas significativos sobre los y las jóvenes.

Frente a la producción revisada en otras regiones del país, para Colombia se analiza el estudio dirigido por Escobar publicado en el año 2004: "La investigación sobre juventud en Colombia: construcción de los sujetos desde los discursos especializados", en la que presenta los antecedentes investigativos sobre los jóvenes en el periodo comprendido entre 1985 y el año 2003. De igual forma, el trabajo de Cabra y Escobar (2014) "El cuerpo en Colombia. Estado del arte cuerpo y subjetividad", sobre el estado de los estudios del cuerpo, presencias y ausencias temáticas en el periodo 19932013, el cual resalta cómo las investigaciones sobre los jóvenes han tomado el cuerpo como categoría que permite comprender las maneras en que estos sujetos han sido incorporados en la sociedad, a través de mecanismos y estrategias de producción o reproducción de una subjetividad particular.

En este mismo contexto se considera la investigación desarrollada por Reina (2012) "Historia de los jóvenes en Colombia 1903-1991", la cual se estructura en cuatro escenarios representativos de la vida nacional: la política, los ejércitos, el estudiantado y la cultura.

Para el caso de América Latina se revisa el estudio realizado por Pérez Islas (2006) "Trazos para un mapa de la investigación sobre juventud en América Latina"; este toma como periodo de indagación los últimos 20 años, basándose en informes regionales, los estados del arte nacionales, las encuestas nacionales de juventud, las publicaciones periódicas especializadas en la materia y los principales grupos de trabajo en este tema. 
Frente a los estudios de RS se analizan diversas investigaciones que se presentan en el desarrollo de este artículo que vinculan este enfoque con el campo educativo y RS del sujeto joven.

El documento da cuenta de las intencionalidades investigativas, los temas de investigación desarrollados, los enfoques y metodologías que han utilizado, los vacíos e inconsistencias, los hallazgos $y$, finalmente, se retoman algunos estudios con enfoque en la teoría de las RS.

\section{Sobre el propósito de las investigaciones}

Martha López (2010), en el estado del arte sobre estudios de jóvenes en Bogotá, se propuso "hacer visible los procesos de resistencia y deseo ligados a la opción cultural de los jóvenes y las jóvenes que actúan en el límite de una cultura excluyente de las diferencias proponiendo vías alternas para una construcción de lo posible" (p. 7). Con base en este estudio, se buscó dar lineamientos de política pública y hacer recomendaciones pertinentes a la inclusión de las prácticas culturales de los jóvenes en los planes de desarrollo de la ciudad.

Según Serrano et al. (2002), muy pocas de las investigaciones pretenden "dar cuenta de los jóvenes escolarizados, de comprender el sistema escolar para aproximarse a las realidades juveniles. Las investigaciones buscan incidir en el espacio escolar para afectar y comprender el mundo juvenil" (p. 48).

Los estudios revisados en Bogotá, Colombia y Latinoamérica pretenden hacer visibles a los jóvenes como sujetos de acción, al dar cuenta de sus prácticas culturales, e incidir en la política pública, indagarlos para comprender las dinámicas de la violencia en las que se les considera protagonistas y causa de muchos de los problemas sociales. Con lo anterior, se busca crear políticas "profilácticas" de intervención.

\section{Conceptos y temas en las}

investigaciones sobre jóvenes

Bogotá distrito capital

Los objetos, temas y conceptos más importantes que se encuentran en los estudios sobre los jóvenes realizados en la ciudad de Bogotá, según los estudios de Serrano et al. (2002) y López (2010) son: percepción sobre los jóvenes en diferentes grupos sociales, es decir, cómo son vistos por otros sujetos; estereotipos que se les asigna; culturas juveniles; participación social y política; imágenes de futuro de los jóvenes y proyectos de vida; educación, formación para el trabajo e inserción socio-laboral; violencia (estudios sobre pandillas y prácticas delincuenciales relacionadas con la juventud y cómo esta violencia los afecta); cuerpo (prácticas de cuidado, comportamiento sexual y reproductivo, género); consumos culturales; políticas públicas de juventud.

Dentro de estos estudios se destacan por su regularidad y mayor frecuencia los de culturas juveniles. Entre estos destacan: Amaya y Marín (2000), García y Giraldo (2000) y Guerrero (1992). También aparecen estudios sobre participación política que se manifiestan por medio de agrupaciones y actividades en torno a lo cultural, a lo cívico-comunitario y a lo político, como: Alfonso (1996), Daza (1996), González (2000). En lo concerniente a política pública se encuentra el trabajo de Rodríguez (2000). En lo relacionado con sexualidad: Álvarez y Suárez (1998), Chávez (1995), Idrobo (2000). Y, por último, en cuanto orientación y consumos culturales Paola García (1998) y Olarte (1995), estos como trabajos representativos.

La condición social de los jóvenes tiene bastante injerencia en estas investigaciones y por ello se da mayor énfasis a los jóvenes de sectores marginales en condiciones de pobreza y exclusión social.

Para Serrano et al. (2002), las investigaciones se dan en torno a temas como "las identidades, las culturas, las estéticas, las sensibilidades y en general aquello que particulariza al joven de otros sujetos sociales y que no se reduce a una variable demográfica como la edad" (p. 90). El tema de la globalización y cómo incide en la vida de los jóvenes es recurrente "la afectación de estas lógicas sobre la vida y la experiencia de la juventud en nuestro medio, auscultan el modo como ellas y ellos enfrentan la situación en la vida cotidiana, haciendo énfasis en las vías alternas que proponen" (López, 2010, p. 9).

Se indaga sobre la pobreza, los agenciamientos y lógicas globales del modelo neoliberal; el modo 
en que a través de las propuestas culturales y artísticas los grupos de jóvenes construyen prácticas identitarias que contribuyen al cambio social en la perspectiva de la inclusión y la diferencia.

Los temas de algunos estudios en los últimos años se relacionan con el cuerpo, el cual se mira hoy como "territorio donde se ejercen fuerzas y se producen mutaciones" (López, 2010, p. 55). En estas temáticas cobran relevancia el cuerpo y su relación con la construcción identitaria, así como los estudios de la subjetividad.

En menor proporción se encuentran los estudios que abordan el poder de los medios masivos de comunicación y la construcción de la vida público-privada en la juventud; además del vínculo entre comunicación y juventud y la resistencia vista como creación artística. En este contexto las expresiones musicales tienen un lugar central; temas como el rock, rap, la radio, el toque, el "pogo" vinculados a la sensibilidad y conflictos juveniles tienen un lugar importante.

En estas investigaciones se mencionan de manera escasa estudios que abordan la postura de los jóvenes desde la teoría de las RS. En este campo se encuentra la investigación de Hernández (2012) sobre las RS que tiene un grupo de jóvenes con respecto a la participación; esta investigación se aborda desde un paradigma cualitativo y que asume el enfoque estructural en el estudio de las RS.

\section{Otras regiones del país}

Las investigaciones desarrolladas en Colombia abordan en su mayoría los mismos temas que los revisados para la ciudad de Bogotá: culturas juveniles, consumos culturales y el cuerpo. Sin embargo, lo encontrado por Escobar (2004) muestra la importancia que asumen los estudios sobre participación social y política durante la década de 1990.

Para Escobar (2004):

[...] se pueden rastrear en estas investigaciones las condiciones que hacen a los jóvenes parte de las formas de producción-reproducción de un sistema social [...] las circunstancias materiales en las que se es joven hoy, en las diferentes regiones de un país como Colombia y en el marco de un modelo neoliberal de globalización. (p. 10)
Para el autor, ciertas investigaciones en Colombia asumen como temas: nociones de sujeto (vulnerabilidad y riesgo, peligro social, búsqueda de identidad, cambio social, entre otros); diversidad (clase social, urbano-rural, género, orientación sexual, étnico-racial y discapacidad).

\section{América Latina}

Para Pérez Islas (2006), en América Latina se instauran los estudios sectoriales: el empleo y los jóvenes, la salud juvenil, la participación política de los jóvenes, jóvenes de poblaciones urbano-marginales, estudiantes de nivel medio, universitarios y proyectos de trabajo con jóvenes. Además de comportamientos juveniles como: la cultura y el deporte, la violencia y las formas participativas en las democracias reinstauradas.

Al analizar el estudio de Pérez Islas se encuentra que son muchos temas los que se abordan en los estudios sobre los jóvenes en América Latina a partir de la década de 1970: educación; salud; empleo; migración; el rock y las nuevas formas de expresión y representación juvenil; las relaciones de los jóvenes con sus familias; la pobreza y cómo afecta a la población juvenil según su sexo, región (rural o urbana y tipo de hogar); consumos culturales; juventud fronteriza; comunicación; recreación y tiempo libre; mujer joven; bandas juveniles; juventud rural y participación política de los jóvenes; cultura juvenil y medios; valores y religión en los jóvenes; sexualidad juvenil; juventud y adicciones; formas de agregación juvenil.

Las investigaciones desarrolladas por Valenzuela y González (1999) posicionan los estudios sobre identidades o identificaciones juveniles y la historia cultural de la juventud dentro del ámbito cultural: manifestaciones ligadas a la música, en las que el rock se asume como tema central.

Para Pérez Islas (2006), en América Latina en los últimos tiempos se destacan estudios sobre "las transformaciones tecnológicas y sus impactos en las nuevas generaciones (particularmente la informática e Internet)" (p. 152), así como el consumo de nuevos tipos de drogas y el sida.

Recientemente aparece una línea de investigación en México, con investigadores como Eduardo Weiss (2012) o Guzmán y Saucedo (2005), y en 
Brasil con Juarez Dayrell, que estudian a los jóvenes en vínculo con la escuela en los que posicionan al estudiante como joven a través de categorías como jóvenes y bachillerato, subjetividad, socialidad y socialización.

\section{Sobre la ruta: enfoques y metodología \\ Paradigmas y enfoques de investigación}

Las investigaciones sobre jóvenes en Colombia y América Latina a partir de los estudios revisados privilegian los paradigmas cualitativos frente a los métodos cuantitativos y mixtos, los cuales se encuentran en menor grado. Los estudios cuantitativos son más evidentes en campos disciplinares como la salud; los diseños experimentales, cuasi-experimentales, ex post facto, con grupos de control, y estudios de caso se dan en investigaciones del campo de la educación.

Las metodologías a través de las cuales se abordan las pesquisas son: las historias de vida, historias orales, testimonios directos de los jóvenes, grupos focales y enfoques etnográficos. En concordancia con los pocos estudios en paradigmas cuantitativos, las encuestas no son instrumentos relevantes en las investigaciones.

Dentro de los estudios etnográficos el método más común es la observación participante en los contextos de interacción cotidiana de los jóvenes.

En investigaciones que indagan por las percepciones y estereotipos sobre los jóvenes se privilegian instrumentos como las entrevistas y las encuestas a los adultos que se relacionan con ellos.

En coherencia con los paradigmas, enfoques y métodos, para el análisis de la información se da prelación al análisis de contenido a partir de categorías preestablecidas. En el proceso analítico muchas investigaciones en este campo usan software de análisis de información documental. Paquetes informáticos como Epi-Info y SPSS (programas para manejar datos en formato de cuestionario y estadísticos en estudios que manejan mucha información), Ethnograph (para el análisis descriptivo interpretativo de textos), NUD*IST (programa para el análisis de datos cualitativos) y Atlas-Ti (en investigaciones con muchos datos), como en el estudio de Serrano et al. (2002), en el que recopiló información de 18 ciudades capitales, para un periodo de 20 años, sobre jóvenes con una muestra de 2457 títulos, el cual se analiza en este artículo.

Según Serrano et al., los procesos de interpretación en las investigaciones recurren a enfoques fenomenológicos, hermenéuticos, histórico-hermenéuticos y etnográficos, así como a estudios de carácter exploratorio y descriptivo.

Dentro del enfoque histórico se destaca la investigación realizada por Reina (2012), en la cual recoge el protagonismo histórico de las juventudes colombianas en el periodo 1903-1991, desde la perspectiva de la construcción de una aproximación para una historia de los jóvenes en Colombia.

Para Pérez Islas, en las aproximaciones generales a las investigaciones sobre jóvenes se destacan:

Las orientadas al sujeto (que consideran al fenómeno juvenil a partir de consideraciones individuales y de carácter intrapsicológico) y las orientadas al contexto (que lo estudian a partir de las transformaciones económicas, sociales y culturales propias de la sociedad), con seis perspectivas específicas: demográfica (definición etaria y tratamiento demográfico); ciudadana (jurídica); moratoria (que puede ser de ciudadanía, de identidad, o de exclusión); de modernización (integración o radicalización de la modernidad); crítica (pertenencia a clase social), y generacional (crítica a los modelos adultos). (2006, p. 155)

Frente a los instrumentos utilizados en los procesos de investigación en América Latina se da prioridad a las entrevistas y encuestas.

\section{Vacíos e inconsistencias en los estudios sobre jóvenes}

En cuanto a los vacíos, según Serrano et al., hay:

[...] reducido número de investigaciones en estratos altos, las redundancias en los contextos de "vulnerabilidad", las dificultades al conectar este tipo de información con otros ejes de la investigación social y por ende el poco interés que se registra en la construcción de metodologías de análisis que posibiliten transformaciones. (2002, p. 39)

En términos generales se pueden listar una serie de vacíos e inconsistencias: 
- Hay una mirada del joven como sujeto masculino y poca investigación sobre su vinculación laboral.

- En Colombia, varios estudios presentan metodologías descriptivas, a pesar de tener como referentes principales los enfoques cualitativos y hermenéuticos.

- No se privilegian los enfoques históricos culturales que den razón de las particularidades regionales, por tanto, no tienen en cuenta las características del entorno cultural.

- Según Pérez Islas, en lo concerniente a América Latina, varias investigaciones se centran en la relación educación mercado laboral y están dirigidas a la elaboración y evaluación de políticas públicas.

- En la región "sigue predominando un enfoque del riesgo, que se conjuga entendiéndola como juventud peligrosa" (Pérez, 2006, p. 155) violencia, delincuencia, drogas y sexualidad, los cuales asumen como población de estudio a la juventud urbana.

- Los estudios centrados en metodologías definidas desde teorías de investigación social como las RS para el estudio de los jóvenes son escasos.

\section{Hallazgos}

En el estudio de Serrano et al. (2002) se describe una visión contradictoria frente a la percepción del sujeto joven, en primer lugar, una mirada negativa, pues está relacionada con: violencia, rebeldía, antipatía, trasgresión, con el mercado, el consumo de sustancias, carente de identidad, vulnerable y sin capacidad necesaria para tomar decisiones acertadas. Lo anterior termina enfrascando al sujeto joven en unos modelos del ser joven basados en el consumo de mercancías: "como carentes de juicio e incapacitados para razonar frente a su propio desarrollo afectivo, cognitivo y físico" (Salazar et al., 1998).

Por otro lado, se encuentra una mirada positiva en la que los jóvenes son percibidos con capacidades de transformación de la sociedad. Los estudios de Perea (2000) presentan "un imaginario del joven como presencia movilizadora de propuestas de futuro". En esta misma vía, López (2010) encuentra en algunos estudios que "las prácticas culturales de los/as jóvenes involucran alternativas identitarias y subjetividades novedosas que señalan hacia nuevas éticas, y éstas permiten construirse un cuerpo, proveerse una mente y dar lugar a un mundo posible" (p. 10).
Para Escobar, algunas investigaciones sitúan al joven como portador de sentidos, pues el "reconocimiento de su capacidad de producir cultura, concretamente es la noción de un joven portador de una cultura específica (subcultura, micro culturas, culturas juveniles, etc.). Aquí el sujeto es en el presente, se aborda su potencia creadora de sentidos y prácticas" (2004, p. 12). Desafortunadamente, son muy pocas las investigaciones que asumen esta mirada.

Hernández (2012) asume al joven como sujeto de acción, frente a la percepción tradicional que se tiene al respecto "la construcción de los diversos discursos sobre los hombres y mujeres jóvenes y sobre la subjetividad juvenil, han estado muy distantes de reconocerlos como sujetos de acción capaces de construir y transformar la realidad social" (p. 55). Visión que es retomada también por Delgado y Arias (2008), Useche (2009), Amador et al. (2011) y Muñoz (2011). Estos autores invitan a entender a los jóvenes más allá de las visiones homogéneas y hegemónicas que se ha construido sobre ellos.

Otros estudios como los referenciados por Useche (2009), muestran cómo los medios han presentado una imagen juvenil de sujetos gozosos, rumberos, que siempre están felices porque la vida les sonríe, porque consumen, porque siguen las tendencias de la moda, porque son deportivos, porque son bonitos.

Desde hace más de una década empezaron a hacer carrera los estudios sobre las subjetividades referidas a las formas, modos de ser y estar en el mundo, de modo que se sitúa al joven como sujeto de derechos.

Serrano et al. relatan las diversas miradas sobre el joven: al joven de los sectores populares se le relaciona con la idea de ser un sujeto en alto riesgo, lo que obliga la realización de programas de prevención, así como una visión que pone más énfasis en el futuro de los jóvenes y menos en el presente. Del mismo modo, se relaciona la juventud con una etapa de tránsito a la adultez.

Visto desde la institucionalidad, el joven aparece como un sujeto pasivo que puede ser indagado y buscado desde este lugar para que pueda aportar y participar en el proceso de política pública. 
La principal causa de las problemáticas de los jóvenes se asocia con factores estructurales, sociales y económicos. Por otro lado, la mirada negativa que sobre ellos se cierne es causa de los estereotipos que se transmiten a través de los medios de comunicación. La escuela, según López (2010), refrenda estas percepciones "mediante la hegemonía de la heterosexualidad obligatoria que funciona en todo el campo social" (p. 10).

Las investigaciones muestran la crisis de la educación en tanto que se presenta una desarticulación entre el mundo de los jóvenes y la cultura escolar. Los contenidos escolares asumen una lógica disciplinar muy distante de perspectivas fenomenológicas que se acerque a la realidad y experiencias de estos sujetos, quienes desde su trayectoria escolar se la representan como aburrida pues las tareas escolares no tienen nada que ver con ellos y con su vida cotidiana.

Es importante reiterar que las pesquisas sobre jóvenes en Colombia son recientes. Tal como lo encuentra Escobar, es en la década de 1990 cuando el tema entró en el escenario del conocimiento académico a partir de la pregunta por la violencia; en la década del 2000 se centra en el tema de pandiIlas juveniles; hoy empiezan a desarrollarse investigaciones relacionadas con el desplazamiento y la vinculación de los jóvenes en el conflicto armado.

Escobar (2004) presenta unas tablas que permiten visualizar el derrotero que han seguido los estudios de juventud en Colombia.

Tabla 1. Documentos reseñados según eje temático prioritario

\begin{tabular}{|l|c|c|}
\hline \multicolumn{1}{|c|}{ Eje temático prioritario } & $\begin{array}{c}\text { N. }{ }^{\circ} \text { de } \\
\text { documentos }\end{array}$ & $\%$ \\
\hline Convivencia y conflicto & 59 & 11.6 \\
\hline Cuerpo & 99 & 19.5 \\
\hline $\begin{array}{l}\text { Culturas juveniles/producción } \\
\text { cultural }\end{array}$ & 91 & 17.9 \\
\hline Educación & 70 & 13.8 \\
\hline Familia & 30 & 5.9 \\
\hline Inserción socio-laboral & 16 & 3.2 \\
\hline Participación social y política & 77 & 15.2 \\
\hline Políticas públicas & 25 & 4.9 \\
\hline Visiones de futuro & 27 & 5.3 \\
\hline Sin información & 13 & 2.6 \\
\hline Total & 507 & 100 \\
\hline
\end{tabular}

Fuente: Escobar (2004, p. 32).
La tabla muestra los temas más representativos estudiados en Colombia. Se aprecia que las investigaciones en el contexto educativo no son las más importantes. Sin embargo, presentan un buen número.

Frente a las percepciones o nociones de sujeto joven identificadas en los estudios, como se ha dicho, los jóvenes son identificados en términos de vulnerabilidad y riesgo, carentes de identidad.

Tabla 2. Nociones de Sujeto presentes en los documentos

\begin{tabular}{|l|c|c|}
\hline \multicolumn{1}{|c|}{ Noción de sujeto } & N. ${ }^{\circ}$ documentos & $(\%)$ \\
\hline Vulnerabilidad y riesgo & 177 & 27.5 \\
\hline Peligro social & 64 & 10.0 \\
\hline Cambio social & 114 & 17.7 \\
\hline Búsqueda de identidad & 159 & 24.7 \\
\hline Culturas juveniles & 91 & 14.2 \\
\hline Otros & 38 & 5.9 \\
\hline Total & 643 & 100 \\
\hline
\end{tabular}

Fuente: Escobar (2004, p. 35).

Los estudios en América Latina empiezan a 191 emerger y a multiplicarse a partir del primer año de la juventud en 1985. Sin embargo, se siguen mirando a estos sujetos desde una perspectiva etaria, fisiológica y demográfica. En muchos países de la región se ve al joven como principal protagonista de cambio, quizá por la situación política que vivieron muchos países latinoamericanos. En este sentido, se ve a los jóvenes como principales protagonistas del cambio y actores sociales.

\section{Estudios con enfoque en las RS}

En la revisión hecha hasta el momento no se encuentran investigaciones centradas en las RS que tienen otros sujetos sobre los jóvenes en su condición escolar.

En la indagación para este estudio se encontró la investigación "Representaciones sociales sobre el adolescente entre un grupo de profesionales dedicados a la salud: ¿una cuestión de abordaje disciplinar?", investigación desarrollada por Verónica Tobeña y publicada por Flacso Argentina. El propósito de este trabajo fue indagar por las representaciones sobre el adolescente en profesionales de disciplinas sociales dedicados a la salud (como 
médicos). Se partió de reconocer que la representación que se tiene de los adolescentes incide en la forma en que se interactúa con estos sujetos; esta investigación asumió un enfoque cualitativo exploratorio y descriptivo y escogió una muestra de 23 profesionales. La categoría central del estudio fue: la adolescencia como una categoría sociológica. Como resultados se obtuvo que pocos profesionales representan a los adolescentes como un grupo etario, en condiciones de carencia, vulnerable, como una etapa de riesgo, a que hay que enseñarle a ser responsable y a ejercer la ciudadanía; de otra parte, un grupo mayoritario de los sujetos los representa desde una perspectiva optimista, como símbolo de oportunidades pues es en una etapa de definiciones. En tal sentido, esta representación de adolescente lo ubica como una etapa camino de la adultez.

Como vacío en esta investigación se encuentra que hay una definición del adolescente desde una perspectiva etaria y biológica. Aunque asume la teoría de la representación social para el estudio, la indagación se queda solamente en el campo de la información. Es decir, se centra en el conocimiento que tiene este grupo de profesionales respecto del objeto de representación que son los adolescentes, pero falta la indagación de otros componentes de la representación social como el campo representacional, la actitud y las condiciones de producción de las RS.

La mayoría de las investigaciones relacionadas con los jóvenes se enmarcan en el contexto de las corrientes interaccionistas, específicamente en los estudios de representaciones mutuas de profesores y estudiantes. En este contexto se encuentran los estudios de Silva (2000); Stubbs y Dalamont (1978); Hargreaves (1986), estudio que indaga sobre las expectativas del profesor relacionadas con las exigencias del currículo y la tarea docente; Coll y Miras (1993) y los estudios de Gilly (1980 y 1993) que se referencian en la mayoría de las investigaciones sobre RS en el campo de la educación.

Los estudios de Gilly (1980) arrojan como haIlazgos que las RS que construyen los maestros de los estudiantes tienen una fuerte influencia de los lineamientos normativos que impone la institucionalidad escolar, los objetivos que persigue la escuela, el estudiante ideal y el grado de correspondencia en los resultados de los alumnos con estas exigencias.

Castillo, en su investigación "representación social del rol docente o del joven popular como alumno", retoma a Gilly para referirse a la influencia de los estereotipos en los procesos educativos de los alumnos "el 'estereotipo' toma una existencia autónoma e interviene como factor central en la manera de 'aprehender' al otro, al cual son imputadas las características generales atribuidas a su grupo social de pertenencia" (Castillo, 2006, p. 16).

Para Castillo, es Gilly (1980) quien aporta algunas referencias para aproximarse al objeto de estudio sobre las RS que tienen los profesores sobre los alumnos, estableciendo tres dimensiones: "las condiciones ligadas a la propia historia personal del docente; las condiciones que incluyen al alumno y a la experiencia concreta de relaciones en la institución escolar; y desde una perspectiva más amplia las condiciones normativas generales de la sociedad" (Castillo, 2006, p. 18).

Los estudios sobre RS indagan fundamentalmente sobre la relación que existe entre fracaso escolar con las RS que tienen los profesores sobre los alumnos y los temas de desigualdad social en la escuela a partir del origen social de los alumnos. En esta línea se encuentran trabajos como el de Castillo (2006). Este estudio analiza las repercusiones que tienen las RS de los profesores sobre los alumnos en contextos de pobreza en el proceso educativo en los liceos de Chile. A su vez, asume un enfoque interaccionista en el estudio para dar cuenta de la desigualdad social en estas instituciones a partir de la interacción social que ocurre en las escuelas.

Para Castillo (2006), las investigaciones desde este enfoque (interaccionista) "se centran principalmente en la 'relación pedagógica', en la relación existente entre el 'mundo adulto escolar' y el 'mundo juvenil de los alumnos'" (p. 14).

Estas orientaciones dan pie para pensar la importancia de los enfoques interaccionistas para estudiar las RS sobre los jóvenes, dando prioridad a la relación existente entre el mundo adulto escolar de los profesores y el mundo juvenil de los alumnos y haciendo algunas aproximaciones por la relación pedagógica. 
Según Castillo (2006), desde este enfoque "el proceso educativo se produce a través de la interacción social que acontece entre los alumnos y sus profesores, en una 'experiencia' interpersonal donde surgen expectativas, percepciones y prácticas que son parte constitutiva del proceso educativo" (p. 15).

En los aportes de la investigación de Castillo se muestra cómo, tanto en la construcción de las evaluaciones que se hacen de los estudiantes como en las percepciones que construyen los profesores sobre ellos, existen variables externas principalmente de orden social y existe la influencia de las expectativas y los prejuicios que tienen los profesores en los resultados de estos sujetos.

En el mismo orden, se encuentran los estudios de Rosenthal y Jacobson que se recogen en el libro Pigmalión en la escuela expectativas del maestro y desarrollo intelectual del alumno, en el cual se plantea que los resultados de los alumnos están influenciados por las expectativas de sus profesores: "antes incluso que un profesor haya observado a un alumno en su desempeño escolar, ya tiene un prejuicio sobre su comportamiento" (Rosenthal y Jacobson, 1980, p. 18). Asumen como metodología experimentos estadísticos y estudios de casos. Uno de los hallazgos más importantes en los estudios de estos autores es que la información, conocimientos o prejuicios que tienen los profesores sobre los estudiantes tienen un efecto en los procesos escolares.

El trabajo de Silva (2000) arroja como hallazgos en el estudio de las RS en el campo de la educación, a partir de sus indagaciones previas, que estos: presentan como temas de análisis "las formas de pensar, sentir y actuar de los diferentes tipos de actores involucrados en la trama educativa" (p. 60); e indagan sobre la cotidianidad escolar "como práctica socio-cultural históricamente producida, analizándola no solamente como actividad concreta, sino también como practica pensada" (p. 60).

Como resultado del análisis de tesis e investigaciones de maestría referidas a representaciones sociales, Silva (2000) encuentra que muchos de estos se quedan cortos a la hora de abordar este enfoque teórico y metodológico:
[...] a pesar de hacer referencia a la noción de representación social no le concedían un lugar central en las investigaciones, o abordaban solamente ciertos aspectos y ciertas manifestaciones de las representaciones sociales, o las evocaban tan sólo como factores o determinantes subyacentes para explicar los resultados que trataban de hechos que no tenían en sí mismos estatus de representaciones sociales. (p. 60)

En los estudios sobre RS en el contexto educativo se encuentran investigaciones que indagan sobre representaciones que construyen los alumnos sobre aspectos u objetos de la cotidianidad escolar, no relacionados con representaciones mutuas de los actores educativos. En especial, se encuentra la investigación de Covarrubias y Martínez (2007), en la cual analizan las RS que construyen los estudiantes en la universidad sobre conceptos como el aprendizaje significativo, los significados que los estudiantes le asignan al aprendizaje y los factores que lo promueven.

La investigación de Martínez asume un paradigma cualitativo e interpretativo; es un estudio de caso y de micronivel, en tanto "el interés fue incursionar en el ámbito de las prácticas cotidianas de los sujetos y sus interacciones, sin ninguna pretensión de generalizar las conclusiones obtenidas (Heras, 1997, citado en Covarrubias y Martínez, 2007, p. 55). Por tanto, se interesa en dar importancia al contexto sociocultural de los actores en el espacio escolar.

En este estudio se asigna un rol preponderante al papel del maestro como mediador entre el currículo y la práctica pedagógica. Sin embargo, el alumno no asume un rol importante.

Covarrubias y Martínez presentan un estado del arte sobre investigaciones en RS y educación:

Sobresalen aquellas que se refieren al pensamiento del profesor; investigaciones que recogen sus creencias, expectativas, teorías implícitas y en general sus concepciones relacionadas con la práctica pedagógica, apoyándose en diversas metodologías para atender más allá de sus comportamientos manifiestos u observables. (Covarrubias y Martínez, 2007, p. 50) 
Para justificar la mirada del estudiante sobre sus propias representaciones, las autoras referencian otros estudios que toman este enfoque y dan al estudiante un lugar activo en el proceso educativo:

[...] autores (Stubbs y Dalamont, 1978; Hargreaves, 1986; Coll y Miras, 1993) analizan en sus investigaciones las concepciones que tienen tanto profesores como alumnos de sus propios papeles y las expectativas de unos y otros con respecto a éstos, y a partir de los procesos de categorización y selección mutua muestran que la mediación no es sólo por parte del profesor sino que los alumnos también participan. (Covarrubias y Martínez, 2007, p. 54)

Las autoras muestran los temas de interés de un conjunto de investigaciones sobre RS en el campo educativo las cuales usan como referencia:

Estas examinan las concepciones de los alumnos sobre las estrategias de aprendizaje utilizadas en clase y su relación con las prácticas educativas de los docentes [...]; analizan el pensamiento pedagógico de estudiantes según lo han construido en las relaciones con sus profesores [...]; resaltan la importancia del conocimiento del sentido común y sus respectivas representaciones sociales que guían las prácticas sociales de los actores en los diversos espacios de la vida escolar [...]; describen y analizan las representaciones de estudiantes y de profesores sobre la excelencia escolar que, como tales, guían las prácticas de los agentes que día a día participan en los procesos escolares [...]; analizan las representaciones de estudiantes sobre la interacción con sus profesores, y cómo éstos atribuyen su aprendizaje a diferentes rasgos, cualidades o actitudes; investigan las percepciones y significados que los estudiantes asignan a las formas de evaluación que sus profesores emplean durante su formación, y cómo éstas influyen en las formas o estrategias de aprendizaje con las que se acercan al conocimiento (Covarrubias y Martínez, 2007, pp. 53-54)

\section{Conclusiones}

Como conclusion se puede enunciar que los estudios sobre los jóvenes son recientes. Se destacan los enfocados a las culturas juveniles y la participación política; las condiciones sociales son un referente, particularmente en los jóvenes de sectores populares, pero hay muy pocos estudios en jóvenes de estratos económicos altos.

Temas como las nociones de sujeto en las que se identifica a los jóvenes como vulnerables y en riesgo, peligro social, búsqueda de identidad y cambio social, son los más recurrentes. Las percepciones del sujeto joven se relacionan con: violencia, con la rebeldía, con la antipatía, con la trasgresión, con el mercado, con el consumo de sustancias, carente de identidad, vulnerable y sin capacidad necesaria para tomar decisiones acertadas. En general, se trata de una visión negativa en contraste con pocas investigaciones en las que se les considera como sujetos activos con capacidades de transformación social. Por ello, son estudiados para la creación de políticas de intervención y la prevención desde un enfoque de riesgo en los que se les entiende como peligrosos.

Ciertas investigaciones dan a conocer cómo los medios han presentado una imagen juvenil como sujetos gozosos y rumberos, que siempre están felices porque la vida les sonríe y con una visión que pone énfasis más en el futuro y menos en el presente mirándolos como una etapa de tránsito a la adultez y como sujeto masculino.

Dentro de los resultados de la investigación documental se puede afirmar que son muy reducidas las investigaciones que asumen al sujeto joven en su condición escolar. En este contexto se le mira desde su papel social como alumno y la mirada de los maestros sobre estos sujetos está determinada por los objetivos institucionales y las condiciones normativas encaminadas al ideal del buen alumno. Los estereotipos y las RS que los maestros construyen sobre los estudiantes inciden en los resultados de los procesos escolares.

Se destacan en las investigaciones los paradigmas cualitativos frente a los métodos cuantitativos y mixtos con metodologías como las historias de vida y orales, testimonios directos de los jóvenes en grupos focales y enfoques etnográficos.

Los procesos analíticos e interpretativos asumen enfoques fenomenológicos y hermenéuticos, de modo que dan prelación al análisis de contenido a partir de categorías preestablecidas. 
Como resultado de esta revisión es importante mencionar que se hace necesario desarrollar investigaciones sobre los jóvenes que asuman enfoques históricos culturales, de tal forma que se sitúe a estos sujetos en sus realidades materiales y subjetivas. Investigaciones que permitan comprender los procesos de construcción social de los jóvenes en su condición escolar, que los aborden como sujetos más allá de su entendimiento como alumno o estudiante, investigaciones que diluciden cómo son representados los jóvenes por sus maestros y cómo inciden estas representaciones en su construcción social y en las prácticas que se dan al interior de la escuela.

\section{Referencias}

Alenzuela, J. M. y González, G. (1999). Oye cómo va, reencuentro del rock tijuanense. Región y Sociedad, 13(21). Recuperado de http://lanic. utexas.edu/project/etext/colson/21/21_r6.pdf

Alfonso, G. (1996). Construir democracia: jóvenes en Ciudad Bolívar asumen reto (tesis inédita de pregrado). Departamento de Ciencia Política, Universidad de los Andes, Bogotá, Colombia.

Álvarez, M. y Suárez, R. (1998). Niños y jóvenes prostituidos. Una visión desde la perspectiva de sus derechos. Bogotá: Procuraduría General de la Nación, Universidad de los Andes, Unicef.

Amador, B. J., García, R. y Leonel, Q. (2011). Jóvenes y derechos en la acción colectiva. Voces y experiencias de organizaciones juveniles en Bogotá. Bogotá: Imprenta Distrital.

Amaya, A. y Marín, M. (2000). Nacidos para la batalla. Nómadas, 13, 64-72.

Cabra, N. A. y Escobar. M. R. (2014). El cuerpo en Colombia. Estado del arte cuerpo y subjetividad. El estado de los estudios del cuerpo, presencias y ausencias temáticas en el periodo 1993-2013. Bogotá: lesco, Idep.

Castillo, J. (2006). ¿Representación institucional del «rol docente» o representación del «joven popular» como alumno? Algunas reflexiones respecto a la tensión sobre la que se fundamenta "el proceso educativo» en la enseñanza secundaria en contextos de pobreza. Ultimadécada, 14(24). Recuperado de http:// www.scielo.cl/scielo.php?
Chávez, M. (1995). ¿Funcionará el discurso sobre sexualidad en los adolescentes? Una visión desde lo religioso y lo castrense (tesis inédita de pregrado). Departamento de Psicología, Universidad de los Andes, Bogotá.

Coll, C. y Miras, M. (1993). La representación mutua profesor/alumno y sus representaciones sobre la enseñanza y el aprendizaje. En C. Coll, J. Palacios y A. Marchesi (eds.), Desarrollo psicológico y educación II. Psicología de la educación (pp. 297-314). Madrid: Alianza.

Covarrubias, P. y Martínez, E. C. (2007). Representaciones de estudiantes universitarios sobre el aprendizaje significativo y las condiciones que lo favorecen. Perfiles Educativos, 29(115), 49-71.

Daza, R. (1996). La política nacional de juventud. Nómadas, 4,114-123.

Delgado, R. y Arias, J. C. (2008). La acción colectiva de los jóvenes y la construcción de ciudadanía. Revista Argentina de Sociología, 6(11), 252-276.

Escobar, M. R. (2004). La investigación sobre juventud en Colombia: construcción de los sujetos desde los discursos especializados. Revista Actualidades Pedagógicas, 48, 9-16.

García, C. (1998). En algún lugar parcharemos. Bogotá: Observatorio de Cultura Urbana, Tercer Mundo Editores.

García, F. M. y Giraldo, J. C. (2000). Ser XY en el siglo XXI. Una mirada a la identidad masculina juvenil (tesis de grado). Departamento de Psicología, Pontificia Universidad Javeriana, Bogotá.

García, P. (1998). Jóvenes y medios: discursos de interacción. Rock, radio e industria musical como hacedores de cultura (tesis inédita de pregrado). Facultad de Comunicación Social, Pontificia Universidad Javeriana, Bogotá.

Gilly, M. (1980). Maestro y alumno roles institucionales y representaciones sociales. París: Puf.

Gilly, M. (1993): Las representaciones sociales en el campo de la educación. En D. Jodelet (ed.), Las representaciones sociales (pp. 68-81). París: Puf. 
González, B. (2000). Casas de la juventud. Espacios para soñar, aprender y construir. Bogotá: Colombia Joven, Ministerio de Educación Nacional, UNDCP Naciones Unidas.

Guerrero, B. (1992). Identidad y cultura: un estudio de caso con jóvenes de Ciudad Bolívar (tesis inédita de pregrado). Departamento de Trabajo Social, Universidad Nacional de Colombia, Bogotá.

Guzmán, C. y Saucedo. C. (2005). La investigación sobre alumnos en México: recuento de una década (1992-2002). En Ducoing, P. (coord.), Sujetos, actores y formación, tomo II (pp. 641832). México, D.F.: Comie, IPN, Grupo Ideograma, Colección La Investigación Educativa en México.

Hargreaves, D. (1986). Las relaciones interpersonales en la educación. Madrid: Narcea.

Hernández. C. E. (2012). Representaciones sociales de la participación en los estudiantes de la mesa local de política educativa de la localidad de los Mártires (tesis de maestría). Maestría en Investigación Social Interdisciplinaria, Universidad Distrital Francisco José de Caldas, Bogotá.

Idrobo, H. (2000). Actitudes respecto a la sexualidad y prácticas sexuales de hombres jóvenes universitarios de las ciudades de Santafé de Bogotá y Quito (tesis de grado). Departamento de Psicología. Universidad de los Andes, Bogotá.

López, M. (2010). Estado del arte para los campos del arte y prácticas culturales para la población de juventud en Bogotá D.C. Bogotá: Universidad Nacional de Colombia, Facultad de Artes.

Muñoz, G. (2011). Prácticas políticas de jóvenes "desde abajo y a la izquierda". En: J. C. Baquiro (ed.), Jóvenes y derechos en la acción colectiva. Voces y experiencias de organizaciones juveniles en Bogotá (pp. 31-71). Bogotá: Imprenta Distrital.

Olarte, R. (1995). Toque y pogo. Jóvenes, música, sensibilidad, historias (tesis de grado). Facultad de Comunicación Social, Pontificia Universidad Javeriana, Bogotá.

Perea, C.M. (2000). De la identidad al conflicto: los estudios de juventud en Bogotá. En J. M. Barbero y F. López (comps.), Cultura y región.
Bogotá: Centro de Estudios Sociales (CES) y Ministerio de Cultura.

Pérez, J. A. (2006). Trazos para un mapa de la investigación sobre juventud en América Latina. Papers Revista de sociología, 79, 145-170.

Reina, C. (2012). Historia de los jóvenes en Colombia 1903-1991. Bogotá: Universidad Nacional de Colombia.

Rodríguez, E. (2000). Juventud y políticas públicas en América Latina. Experiencias y desafíos desde la gestión institucional. En Umbrales. Cambios culturales, desafíos nacionales y juventud (pp. 233-268). Medellín: Corporación Región.

Rosenthal, R. y Jacobson, L. (1980). Pygmalion en la escuela. Expectativas del maestro y desarrollo intelectual del alumno. México D.F.: Marova.

Salazar, A. (1998). Juventud, política social y violencia urbana en Santafé de Bogotá. Bogotá: Ministerio de Justicia y del Derecho, Dirección General de Prevención y Conciliación.

Serrano, A. J., Bejarano, R., Caicedo, F. A., Hoyos, G. D. y Quintero, T. F. (2002). Estado del arte de la investigación sobre juventud para la formulación de la política. Bogotá: Departamento Administrativo de Acción Comunal del distrito, departamento de investigaciones, Universidad Central.

Silva, L. (2000). Escuela: espacio intersubjetivo de representaciones múltiples. En Casado E. y Calonge S., Representaciones sociales y educación (pp. 59-72). Cuadernos de posgrado 25. Venezuela.

Stubbs, M. y Dalamont, S. (1978). Las relaciones profesor-alumno. Barcelona: Oikos-Tau.

Tobeña, V. (2005). Representaciones sociales sobre el adolescente entre un grupo de profesionales dedicados a la salud: ¿una cuestión de abordaje disciplinar? Revista Argentina de Sociología, 3(5), 107-132.

Useche, O. (2009). Jóvenes produciendo sociedad, subjetividades, derechos sociales y productividad juvenil. Bogotá: Corporación Universitaria Minuto de Dios.

Weiss, E. (coord.) (2012). Jóvenes y bachillerato. México: Asociación Nacional de Universidades e Instituciones de Educación Superior. 